\title{
Protective Effect of Diphenhydramine against Traumatic Brain Injury in Rats via Modulation of Oxidative Stress and Inflammation
}

\author{
Wenyong Pan ${ }^{a}$ Zhigang Cao $^{a}$ Dongyang Liu $^{b}$ Yingbin Jiao ${ }^{c}$ \\ ${ }^{a}$ Department of Neurosurgery, Qingdao Chengyang People's Hospital, Qingdao, China; ${ }^{b}$ Department of Emergency

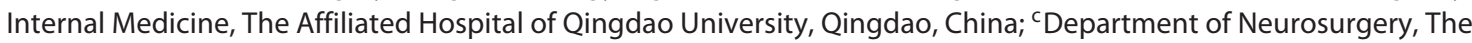 \\ Affiliated Hospital of Qingdao University, Qingdao, China
}

\section{Keywords}

Traumatic brain injury · Neuronal degeneration · Oxidative stress $\cdot$ Inflammation $\cdot$ Apoptosis

\begin{abstract}
Background: Traumatic brain injury (TBI) is considered a major burden across the globe affecting both individuals and their families. Therefore, the present study was conducted to determine the protective effect of diphenhydramine (DPM) against TBI in experimental rats. Methods: The effect of DPM was evaluated on the cerebral edema (CE) and neuronal degeneration after the induction of experimental brain injury in rats. The effect of DPM was also investigated on the inflammatory cytokines, for example, tumor necrosis factor-a and interleukin $1 \beta$ and oxidative stress markers, such as malondialdehyde, superoxide dismutase, and glutathione peroxidase. Western blot analysis was used to investigate the effect of DPM on B-cell lymphoma 2 (Bcl-2), Bcl-2-associated X protein (Bax) and cleaved caspase-3. Results: Results of the study suggest that DPM causes reduction in CE and prevents neuronal degeneration. It also causes reduction in inflammation and oxidative stress in a dose-dependent manner. The level of Bax was found to be elevated, together with reduction in the Bcl-2 level in the DPM-treated group. Conclu-
\end{abstract}

\section{KARGER}

() 2019 S. Karger AG, Basel

E-Mail karger@karger.com

www.karger.com/pha sion: DPM exerts a neuroprotective effect after TBI via the attenuation of oxidative stress, inflammation, and mitochondrial apoptosis pathways.

(c) 2019 S. Karger AG, Basel

\section{Introduction}

Traumatic brain injury (TBI) is considered a major burden across the globe [1-3]. It is reported to have high morbidity and mortality together with higher healthcare costs. The TBI involves highly multifaceted series of events classified as primary and secondary injuries, which leads to either temporary or permanent damage to the brain. The progression of primary injury of TBI to the secondary injury is the main determinant to assess the prognosis of TBI [4-8]. Various studies reported that excessive oxidative stress, generation of free radicals, and inflammatory conditions, which promote neuronal deterioration and apoptosis are classified as the key reasons for the permanent damage of the brain after progression to secondary injury [8-10]. Therefore, if not controlled or treated early, the outcome of secondary injury is lifethreatening and often fatal. In the past decades, various successful attempts have been made to control the pro- 
gression of TBI injuries, or to limit its deleterious effects [11-14]. However, none of these methods are proved to be effective to prevent or limit the damage caused by TBI because of its multifactorial etiology. Thus, the discovery of novel agents, which can act via multiple mechanisms could serve the purpose and provide beneficial effect against TBI.

Diphenhydramine (DPM) is a first-generation $\mathrm{H} 1$ antihistaminic agent used to treat allergic conditions by limiting the release of histamine [15]. It showed a diverse array of pharmacological properties, for instance, sedative [16], anticancer [17], antiemetic [18], local anesthetic [19], and anti-cholinergic activity [20]. DPM is also known for significant antioxidant activity [21]. Therefore, in the present study, we would like to assess the protective effect of DPM against TBI in the light of reported antioxidant and anti-inflammatory activities.

\section{Material and Method}

\section{Chemical and Reagents}

Unless otherwise stated, all chemical and reagents used in the present study were obtained from Sigma Aldrich, USA.

\section{Animals}

ICR mice weighing approximately $26-30 \mathrm{~g}$ were obtained from the institutional animal house and housed in polypropylene cages. They were provided food and water ad libitum. The experiments were approved by the Animal Care and Use Committee of the Affiliated Hospital of Qingdao University (AUC/AHQU/2018/034). Experimental protocols were followed with strict adherence to the regulations set forth by the, Experimental Animal Regulation by the National Science and Technology Commission, China for the use of laboratory animals.

\section{Induction of TBI}

TBI in the ICR mice was induced by the Marmarou's weightdrop model. Mice were placed in a stereotaxic frame after the induction of anesthesia with intraperitoneal injection of $3 \%$ pentobarbital sodium (30 mg/kg). The TBI was induced via dropping $200 \mathrm{~g}$ weight on the left anterior frontal area after surgically exposing fasia and skull. The Sham animals received all except, no weight drop.

\section{Experimental Design}

Six groups were formed as follows and each group contains 18 animals each.

Group 1: Sham

Group 2: TBI

Group 3: TBI + vehicle (Veh)

Group 4: TBI + DPM (5 mg/kg)

Group 5: TBI + DPM $(10 \mathrm{mg} / \mathrm{kg})$

Group 6: TBI + DPM (15 mg/kg)
The mice from each group were anesthetized and sacrificed $24 \mathrm{~h}$ post-TBI for biochemical and histological analyses.

\section{Brain Water Content}

The brain was isolated and located on a chilled brain matrix, where the brain stem and cerebellum were separated. The left cerebral hemispheres were removed and harvested, and the wet weight (ww) of each hemisphere was recorded. The dry weight was obtained after drying the sample at $80^{\circ} \mathrm{C}$ for $72 \mathrm{~h}$. Water content was calculated as a percentage as per the following formula: (ww dry weight) $/$ ww $\times 100 \%$.

\section{Neurological Deficit}

Grip test was performed to evaluate the effect on neurological deficit. The mice were placed on the metal wire tied between 2 vertical poles above a foam pad.

Depending on the mouse's ability to remain on the wire, different scores were given for its ability.

Score 0: If the mouse was unable to remain on the wire for $30 \mathrm{~s}$;

Score 1: If the mouse failed to hold on to the wire with both forepaws and hind paws together;

Score 2: If the mouse held on to the wire with both forepaws and hind paws but not the tail;

Score 3: If the mouse used its tail along with both forepaws and hind paws;

Score 4: If the mouse moved along the wire on all 4 paws plus tail;

Score 5: If the mouse that scored 4 points also ambulated down one of the posts used to support the wire.

It was performed in triplicate, and the total value was calculated for each mouse.

\section{Nissl Staining}

Paraffin-embedded sections of the cerebral cortex were stained with cresyl violet. Normal neurons displayed large cell bodies and round nuclei, and were enriched in cytoplasm. In contrast, damaged cells were shrunken with condensed and hyperchromatic nuclei, and dark cytoplasm containing vesicles. Six fields $(400 \times)$ in each section were observed and the mean number in the 6 views was regarded as the data of each section.

\section{Malondialdehyde Content, Superoxide Dismutase, and}

\section{Glutathione Peroxidase Activity}

Levels of malondialdehyde (MDA) content (nmoL/mg), superoxide dismutase (SOD) and glutathione peroxidase (GPx) activity (U/mg protein) were measured using a spectrophotometer (Nanjing Jiancheng Biochemistry Co, China).

\section{Western Blot Assay}

Ten or $12 \%$ SDS-PAGE was carried out to separate the proteins. Afterwards, western transfer was performed with PVDF membranes (Millipore, Bedford, MA, USA). After blocking, the membranes were incubated with each primary antibody (1: 1,000; CST or Abcam, Cambridge, MA, USA) overnight. Afterwards, the corresponding secondary antibodies (1:2,000; Abcam, Cambridge, MA, USA) were incubated for $2 \mathrm{~h}$. Then the protein bands were visualized using enhanced chemiluminescence substrate (Millipore, Billerica, MA, USA) and photo-
48

Pharmacology 2020;105:47-53

DOI: $10.1159 / 000502767$
$\mathrm{Pan} / \mathrm{Cao} / \mathrm{Liu} / \mathrm{Jiao}$ 


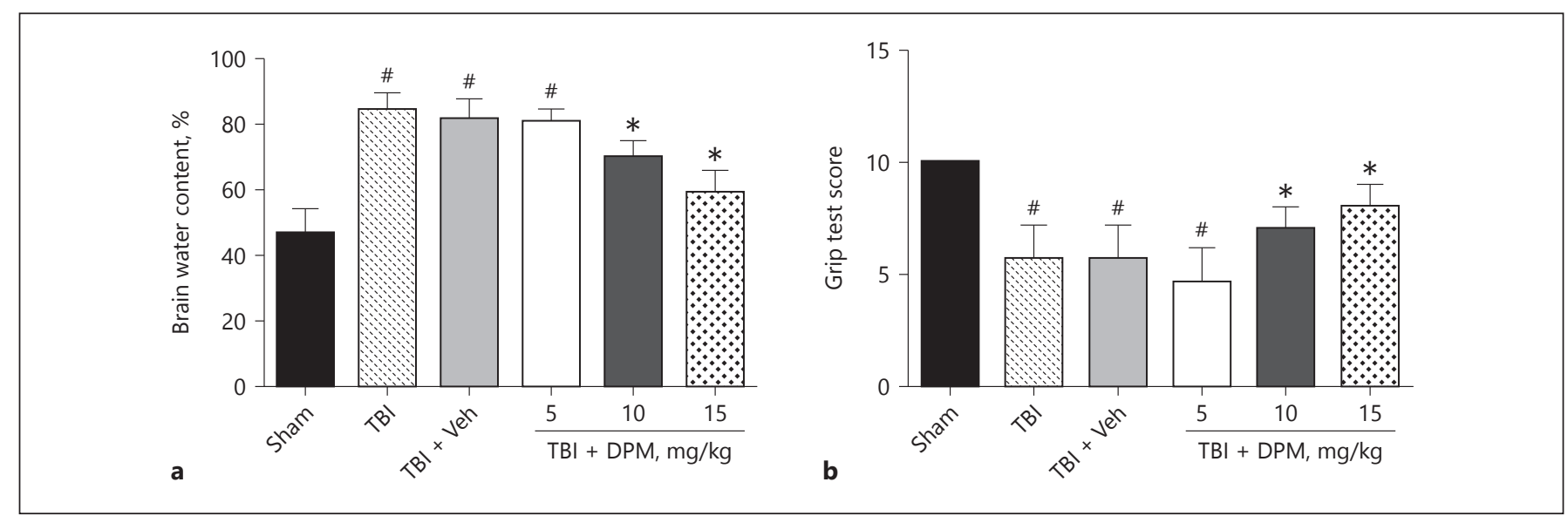

Fig. 1. Effect of DPM on the CE. Values represent the mean \pm SD and are representative of 3 independent experiments. ${ }^{\#} p<0.05$ vs. sham; ${ }^{*} p<0.05$ vs. TBI. TBI, traumatic brain injury; veh, vehicle; DPM, diphenhydramine.

graphed with Image Quant LAS 4000 (Pittsburgh, GE, USA). The grayscale value of interest proteins was analyzed by Image J software (NIH, Bethesda, MD, USA).

Enzyme-Linked Immunosorbent Assay

The determination of tumor necrosis factor- $\alpha$ (TNF- $\alpha$ ), interleukin (IL)-1 $\beta$ was performed using commercially available enzyme-linked immunosorbent assay kits as per manufacturer's instructions.

\section{Result}

\section{Effect of DPM on the Cerebral Edema}

The results of brain water content were enumerated in Figure 1. In the TBI group, the brain water content was found to be significantly elevated, with no considerable difference with TBI after administering Veh. However, in the DPM group, the brain water content was significantly reduced as compared to the TBI + Veh group in a dosedependent manner, with excellent activity in the case of the $15-\mathrm{mg}$ treated group. Thus, $15 \mathrm{mg} / \mathrm{kg}$ was chosen as an optimum dose for further experimentation.

\section{Effect of DPM on the Neuronal Degeneration}

The effect of DPM was determined on the neuronal degeneration of the brain tissues of the experimental mice and the results are presented in Figure 2. The sham group showed the presence of few positive cells. The apoptotic index was found to be enhanced in the TBI and TBI + Veh group as compared to the sham group. A significant decline in the apoptotic index was observed in the DPMtreated group.

Effect of DPM in Traumatic Brain Injury
Western Blot Analysis of DPM Effect on B-Cell

Lymphoma 2 Signaling after TBI

The effect of DPM was then investigated on the neuronal apoptosis via western blot analysis. The expression of cleaved caspase-3 was found to be elevated after TBI, as compared to sham. However, the DPM-treated group showed reduction in the level of cleaved caspse- 3 protein in respect to the TBI + Veh group. The DPM was thought to prevent neuronal degeneration via the attenuation of B-cell lymphoma 2-associated X protein (Bax) translocation. As shown in Figure 3, the level of Bax was found to be increased after TBI, while B-cell lymphoma 2 (Bcl-2) level was found to be decreased, as compared to the Sham group. Nevertheless, in the DPM treated group, the level of Bax was found to be elevated, together with decline in the Bcl-2 level.

\section{Effect of DPM on the Oxidative Stress}

As shown in Figure 4, the level of MDA in mitochondria was found to be elevated in the TBI + Veh group as compared to the sham group, while after administration of DPM, the enhanced level was found to be reduced. On the other hand, DPM also causes the augmentation of the level of GPx and SOD, which is found to be reduced after TBI.

\section{Effect of DPM on Inflammatory Cytokines}

The effect of DPM was quantified on the levels of cytokines in the brains of the experimental mice, and the results are presented in Figure 5. It has been found that the levels of both tested cytokines TNF- $\alpha$ and IL- $1 \beta$ were found to be elevated in the TBI or the TBI + Veh group. 


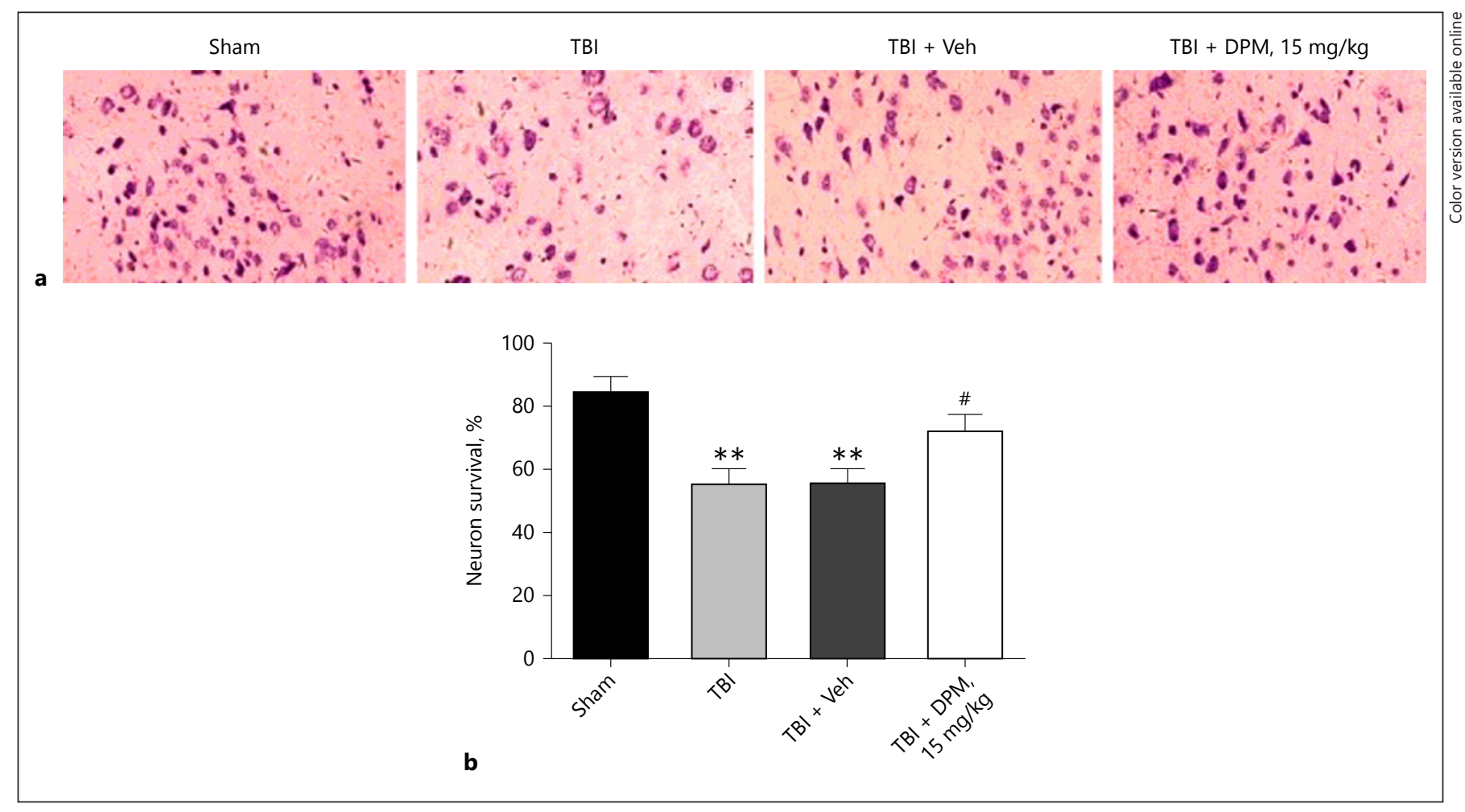

Fig. 2. Effect of DPM on the neuronal degeneration. Values represent the mean \pm SD and are representative of 3 independent experiments. ${ }^{* *} p<0.05$ vs. sham; ${ }^{\#} p<0.05$ vs. TBI. TBI, traumatic brain injury; veh, vehicle; DPM, diphenhydramine.

In the DPM-treated group, the levels of these cytokines were found to be significantly lowered as compared to the TBI group.

\section{Discussion}

TBI is the devastating pathological condition that mainly occurs because of mechanical injury $[22,23]$. Their deleterious effects are as a result of the secondary injury, which progresses from the primary injury. The secondary injury causes neuronal degeneration of the brain via the induction of the inflammatory response and oxidative stress [24-27]. Thus, agents limiting these pathological conditions have proved beneficial against TBI. In the present study, DPM protects the brain after TBI via attenuating oxidative stress, brain edema, and neuronal degeneration.

Following the brain injury, cerebral edema (CE) is the initial consequence where the water level of brain tissues was increased, which not only affects individual cells but also the surrounding interstitial spaces. Various studies have tried to elaborate the pathogenesis of CE in TBI; however, none of the studies have arrived at any conclusive evidence to prove this [28-30]. It could be suggested that, in $\mathrm{CE}$, the integrity of $\mathrm{BBB}$ is lost, together with alteration in various ionic pumps and induction of inflammatory response [31-33]. In the present study, DPM showed the reduction of $\mathrm{CE}$ as evidenced by decrease in brain water content. The improvement of the grip test score further signifies the protective effect of DPM against TBI. TBI-induced neuronal cell death is the major determinant for brain damage. In the present study, DPM showed an improvement of neuronal survival at the tested dose and thus has a protective role against TBI. Apoptosis is the characteristic feature of neuronal cell death after TBI $[5,34,35]$. It is a highly coordinated intracellular event regulated by the activation of various genes and proteins. Among such genes, at the mitochondrial level, the proto-oncogene $\mathrm{Bcl}-2$ controls apoptosis by maintaining an intricate balance between genes which either induce (Bax) or inhibit (Bcl-2) apoptosis [36-38]. Caspase-3 is another vital gene, involved in the repair of DNA and membrane integrity [39-41]. In the present study, DPM showed the downregulation of cleaved caspase- 3 and Bax, 


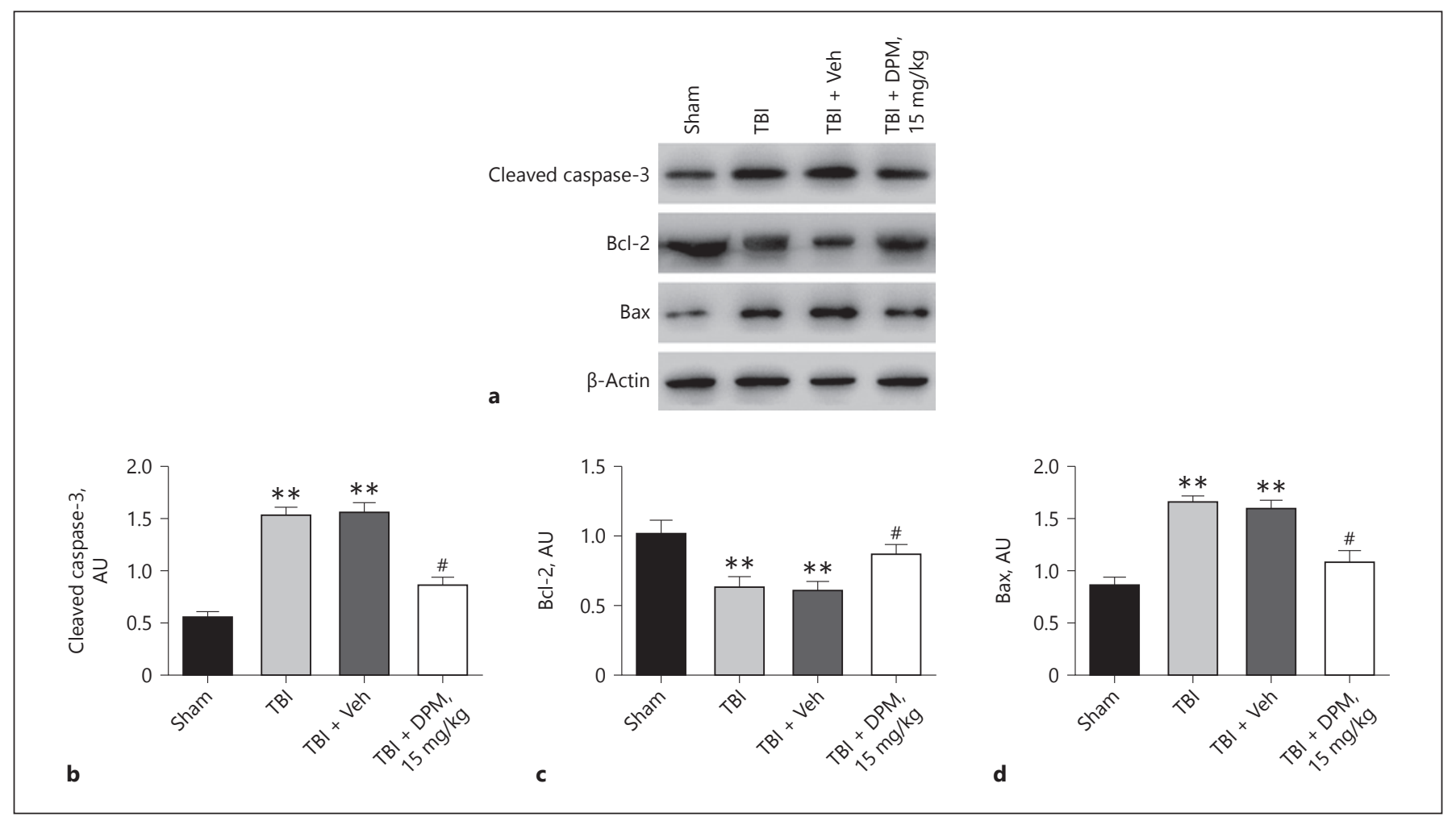

Fig. 3. Effect of DPM on the Bcl-2 family proteins. Values represent the mean \pm SD and are representative of 3 independent experiments. ${ }^{* *} p<0.05$ vs. sham; ${ }^{\#} p<0.05$ vs. TBI. TBI, traumatic brain injury; veh, vehicle; DPM, diphenhydramine; Bcl-2, B-cell lymphoma 2; Bax, Bcl-2-associated X protein.
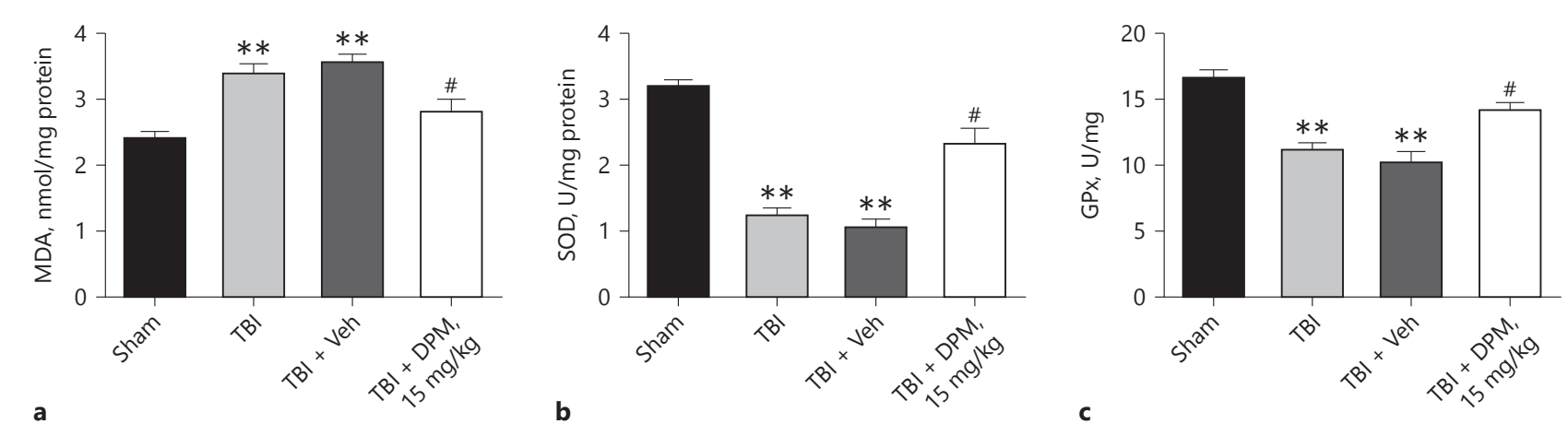

Fig. 4. Effect of DPM on the oxidative stress. Values represent the mean \pm SD and are representative of 3 independent experiments. ${ }^{* *} p<0.05$ vs. sham; ${ }^{*} p<0.05$ vs. TBI. MDA, malondialdehyde; TBI, traumatic brain injury; veh, Vehicle; DPM, diphenhydramine; SOD, superoxide dismutase; GPx, glutathione peroxidase.

with upregulation of Bcl-2 in a dose-dependent manner. The presence of oxidative stress subsequent to TBI via the generation of highly reactive oxygen species is the main destructive factor for the brain damage. This reactive oxygen species triggers the damage of proteins and DNA, and increases membrane permeability via oxidative stress [42, 43]. In the present study, DPM causes a decrease in the oxidative stress as evidenced via reduction in MDA and upregulation of SOD and GPx to near control. These results were further found in agreement with the anti-oxi- 


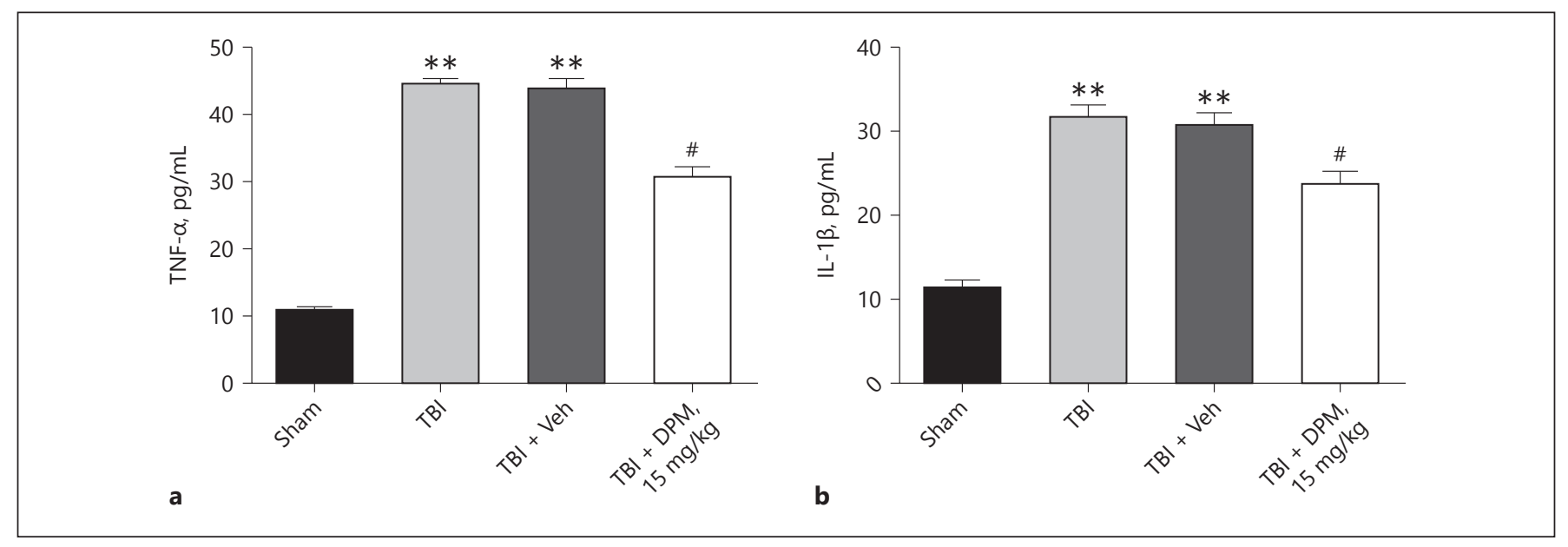

Fig. 5. Effect of DPM on the pro-inflammatory cytokines. Values represent the mean \pm SD and are representative of 3 independent experiments. ${ }^{* *} p<0.05$ vs. sham; ${ }^{\#} p<0.05$ vs. TBI. TNF- $\alpha$, tumor necrosis factor- $\alpha$; TBI, traumatic brain injury; veh, vehicle; DPM, diphenhydramine; IL-1 $\beta$, interleukin $1 \beta$.

dative activity of DPM reported earlier [21]. Inflammation of the brain after mechanical injury is another hallmark of TBI. It activates microglia and astrocytes, with subsequent release of proinflammatory cytokines within the brain (TNF- $\alpha$ and IL-1 $\beta$ ), and recruitment of peripheral immune cells [44-47]. In the present study, DPM causes reduction in the level of both TNF- $\alpha$ and IL- $1 \beta$.

The results of the study concluded that DPM exerts a neuroprotective effect after TBI via the attenuation of ox- idative stress, inflammation, and mitochondrial apoptosis pathways. Thus, more studies are needed to utilize DPM against secondary events of TBI.

\section{Disclosure Statement}

The authors declare that they have no conflicts of interest to disclose.

\section{References}

1 Zaninotto AL, Costa BT, Ferreira IS, French M, Paiva WS, Fregni F. Traumatic brain injury. Neuromethods; 2018. pp. 105-38.

2 Corrigan JD, Selassie AW, Orman JA. The epidemiologyo of traumatic brain injury. J Head Trauma Rehabil. 2010;25(2):72-80.

3 Romner B, Grände PO. Traumatic brain injury: intracranial pressure monitoring in traumatic brain injury. Nat Rev Neurol. 2013; 9(4):185-6.

4 Johnson VE, Stewart W, Smith DH. Axonal pathology in traumatic brain injury. Exp Neurol. 2013;246:35-43.

5 McGinn MJ, Povlishock JT. Pathophysiology of Traumatic Brain Injury. Neurosurg Clin N Am. 2016;27(4):397-407.

6 Mckee AC, Daneshvar DH. The neuropathology of traumatic brain injury; in: Handbook of Clinical Neurology. 2015. vol 127. pp. 4566.

7 Rosenfeld JV, Maas AI, Bragge P, MorgantiKossmann MC, Manley GT, Gruen RL. Early management of severe traumatic brain injury. Lancet. 2012;380(9847):1088-98.
8 Prins M, Greco T, Alexander D, Giza CC. The pathophysiology of traumatic brain injury at a glance. Dis Model Mech. 2013;6(6):130715.

9 Blennow K, Brody DL, Kochanek PM, Levin H, McKee A, Ribbers GM, et al. Traumatic brain injuries. Nat Rev Dis Primers. 2016; 2(1): 16084 .

10 Desai M, Jain A. Neuroprotection in traumatic brain injury. J Neurosurg Sci. 2018;62:56373.

11 Hu ZG, Wang HD, Qiao L, Yan W, Tan QF, Yin HX. The protective effect of the ketogenic diet on traumatic brain injury-induced cell death in juvenile rats. Brain Inj. 2009;23(5): 459-65.

12 Pandit V, Patel N, Rhee P, Kulvatunyou N, Aziz H, Green DJ, et al. Effect of alcohol in traumatic brain injury: is it really protective? J Surg Res. 2014;190(2):634-9.

13 Li X, Wang H, Gao Y, Li L, Tang C, Wen G, et al. Protective effects of quercetin on mitochondrial biogenesis in experimental traumatic brain injury via the $\mathrm{Nrf2}$ signaling pathway. PLoS One. 2016; 11(10):e0164237.

14 Lee JH, Wei L, Gu X, Wei Z, Dix TA, Yu SP. Therapeutic Effects of Pharmacologically Induced Hypothermia against Traumatic Brain Injury in Mice. J Neurotrauma. 2014;31(16): 1417-30.

15 Miller SM, Cumpston KL. Diphenhydramine; in: Encyclopedia of Toxicology: Third Edition. 2014. pp. 195-7.

16 Hofmeister EH, Egger CM. Evaluation of diphenhydramine as a sedative for dogs. J Am Vet Med Assoc. 2005;226(7):1092-4.

17 Or CH, Su HL, Lee WC, Yang SY, Ho C, Chang CC. Diphenhydramine induces melanoma cell apoptosis by suppressing STAT3/ MCL-1 survival signaling and retards B16F10 melanoma growth in vivo. Oncol Rep. 2016;36(6):3465-71.

18 Lin TF, Yeh YC, Yen YH, Wang YP, Lin CJ, Sun WZ. Antiemetic and analgesic-sparing effects of diphenhydramine added to morphine intravenous patient-controlled analgesia. Br J Anaesth. 2005;94(6):835-9. 
19 Pavlidakey PG, Brodell EE, Helms SE. Diphenhydramine as an alternative local anesthetic agent. J Clin Aesthet Dermatol. 2009 Oct:2(10):37-40.

20 Orzechowski RF, Currie DS, Valancius CA. Comparative anticholinergic activities of 10 histamine $\mathrm{H} 1$ receptor antagonists in two functional models. Eur J Pharmacol. 2005; 506(3):257-64.

21 Kesiova M, Alexandrova A, Yordanova N, Kirkova M, Todorov S. Effects of diphenhydramine and famotidine on lipid peroxidation and activities of antioxidant enzymes in different rat tissues. Pharmacol Rep. 2006;58:221-8.

22 Kolias AG, Guilfoyle MR, Helmy A, Allanson J, Hutchinson PJ. Traumatic brain injury in adults. Pract Neurol. 2013;13(4):228-35.

23 Hyder AA, Wunderlich CA, Puvanachandra P, Gururaj G, Kobusingye OC. The impact of traumatic brain injuries: a global perspective. NeuroRehabilitation. 2007;22:341-53.

24 Woodcock T, Morganti-Kossmann MC. The role of markers of inflammation in traumatic brain injury. Front Neurol. 2013 Mar 4.

25 Cornelius C, Crupi R, Calabrese V, Graziano A, Milone P, Pennisi G, et al. Traumatic Brain Injury: Oxidative Stress and Neuroprotection. Antioxid Redox Signal. 2013;19(8):836-53.

26 Quillinan N, Herson PS, Traystman RJ. Neuropathophysiology of Brain Injury. Anesthesiol Clin. 2016;34(3):453-64.

27 Kochanek PM, Jackson TC, Ferguson NM, Carlson SW, Simon DW, Brockman EC, et al. Emerging therapies in traumatic brain injury. Semin Neurol. 2015;35(01):83-100.
28 Unterberg AW, Stover J, Kress B, Kiening KL. Edema and brain trauma. Neuroscience. 2004;129(4):1019-27.

29 Donkin JJ, Vink R. Mechanisms of cerebral edema in traumatic brain injury: therapeutic developments. Curr Opin Neurol. 2010;23(3): 293-9.

30 Raslan A, Bhardwaj A. Medical management of cerebral edema. Neurosurg Focus. 2008; 22(5):1-12.

31 Chodobski A, Zink BJ, Szmydynger-Chodobska J. Blood-Brain Barrier Pathophysiology in Traumatic Brain Injury. Transl Stroke Res. 2011;2(4):492-516.

32 Alves JL. Blood-brain barrier and traumatic brain injury. J Neurosci Res. 2014;92(2):141-

33 Pun PB, Lu J, Moochhala S. Involvement of ROS in BBB dysfunction. Free Radic Res. 2009;43(4):348-64.

34 Peeters W, van den Brande R, Polinder S, Brazinova A, Steyerberg EW, Lingsma HF, et al. Epidemiology of traumatic brain injury in $\mathrm{Eu}$ rope. Acta Neurochir (Wien). 2015;157(10): 1683-96.

35 Raghupathi R, Graham DI, McIntosh TK. Apoptosis After Traumatic Brain Injury. J Neurotrauma. 2000 Oct;17(10):92738.

36 Ruvolo PP, Deng X, May WS. Phosphorylation of Bcl2 and regulation of apoptosis. Leukemia. 2001;15(4):515-22.

37 Zinkel S, Gross A, Yang E. BCL2 family in DNA damage and cell cycle control. Cell Death Differ. 2006;13(8):1351-9.
38 Dai H, Meng W, Kaufmann S. BCL2 Family, Mitochondrial Apoptosis, and Beyond. Cancer Transl Med. 2016;2(1):7.

39 Porter AG, Jänicke RU. Emerging roles of caspase-3 in apoptosis. Cell Death Differ. 1999; 6(2):99-104.

40 Jänicke RU, Sprengart ML, Wati MR, Porter AG. Caspase-3 is required for DNA fragmentation and morphological changes associated with apoptosis. J Biol Chem. 1998;273(16): 9357-60.

41 Wolf BB, Schuler M, Echeverri F, Green DR. Caspase- 3 is the primary activator of apoptotic DNA fragmentation via DNA fragmentation factor-45/inhibitor of caspase-activated DNase inactivation. J Biol Chem. 1999; 274(43):30651-6.

42 Wang Q, Tang XN, Yenari MA. The inflammatory response in stroke. J Neuroimmunol. 2007;184(1-2):53-68.

43 Bains M, Hall ED. Antioxidant therapies in traumatic brain and spinal cord injury. Biochim Biophys Acta Mol Basis Dis. 2012; 1822(5):675-84.

44 Russo MV, McGavern DB. Inflammatory neuroprotection following traumatic brain injury. Science. 2016 Aug 19;353(6301):783-5.

45 Donat CK, Scott G, Gentleman SM, Sastre M. Microglial activation in traumatic brain injury. Front Aging Neurosci. 2017;9:208.

46 Karve IP, Taylor JM, CrackPJ. The contribution of astrocytes and microglia to traumatic brain injury. BrJ Pharmacol. 2016;173(4):692-702.

47 Chen Z, Trapp BD. Microglia and neuroprotection. J Neurochem. 2016;136:10-7. 